\title{
Disruption of Activity in the Ventral Premotor But Not the Anterior Intraparietal Area Interferes with On-Line Correction to a Haptic Perturbation during Grasping
}

\author{
Luis F. Schettino, ${ }^{1 \star}$ Sergei V. Adamovich, ${ }^{2,3 *}$ Hamid Bagce, ${ }^{4,5}$ Mathew Yarossi, ${ }^{3,4}$ and Eugene Tunik ${ }^{3,4}$ \\ ${ }^{1}$ Psychology Department, Lafayette College, Easton, Pennsylvania 18042, ${ }^{2}$ Department of Biomedical Engineering, New Jersey Institute of Technology, \\ Newark, New Jersey 07102, and ${ }^{3}$ Department of Rehabilitation and Movement Science, ${ }^{4}$ Graduate School of Biomedical Engineering, and ${ }^{5}$ New Jersey \\ Medical School, Rutgers University, Newark, New Jersey 07101
}

\begin{abstract}
Replanning ongoing movements following perturbations requires the accurate and immediate estimation of the motor response based on sensory input. Previous studies have used transcranial magnetic stimulation (TMS) in humans to demonstrate the participation of the anterior intraparietal sulcus (aIPS) and ventral premotor cortex (PMv) in visually mediated state estimation for grasping. Here, we test the role of parietofrontal circuits in processing the corrective responses to haptic perturbations of the finger during prehension. Subjects reached to grasp an object while having to compensate for a novel and unpredictable haptic perturbation of finger extension. TMS-based transient disruptions to the PMv and aIPS were delivered 0, 50, or $100 \mathrm{~ms}$ after the perturbation. TMS to the PMv delivered $50 \mathrm{~ms}$ after the perturbation (but not 0 or $100 \mathrm{~ms}$, or in unperturbed trials) led to an overestimation of grasp aperture. No effects on grasp aperture were noted for the aIPS. Our results indicate that the PMv (but not aIPS) is involved in the deployment of the compensatory response in the presence of haptic perturbations during prehension. Our data also identify the time window of neural processing in the PMv when reprogramming occurs to be $50-100 \mathrm{~ms}$ following the perturbation onset.
\end{abstract}

Key words: frontoparietal; grasping; haptic perturbation; on-line control; TMS; updating

\section{Introduction}

The role of parietofrontal circuits in the control of reaching and grasping has been a major focus of investigation (Grafton, 2010). Neurons in the anterior intraparietal area (AIP) of nonhuman primates encode visual features of objects during prehension (Murata et al., 2000). The human homolog of the AIP, the anterior intraparietal sulcus (aIPS; Binkofski et al., 1999; Culham, 2004; Frey et al., 2005) is also responsive to graspable objects, suggesting a role in visually based updating of grasp. Our prior work using transient cortical disruptions via transcranial magnetic stimulation (TMS) confirms this hypothesis, showing that aIPS TMS delays on-line adjustments of grasp aperture when correcting for a perturbed object goal (Tunik et al. 2005).

Empirical data suggest that ventral premotor cortex (PMv) may also be important for prehension. Profuse projections from AIP to PMv (area F5) of the macaque brain have been described

\footnotetext{
Received July 21, 2014; revised Nov. 17, 2014; accepted Dec. 16, 2014

Author contributions: S.V.A. and E.T. designed research; L.F.S., S.V.A., H.B., and E.T. performed research; L.F.S., S.V.A., H.B., and E.T. analyzed data; L.F.S., S.V.A., M.Y., and E.T. wrote the paper.

This work was supported by the National Institute of Neurological Disorders and Stroke under Award R01NS085122 (E.T.) and the National Institute of Child Health and Human Development under Award R01HD58301 (S.V.A.).

*L.F.S. and S.V.A. contributed equally to this work.

The authors declare no competing financial interests.

Correspondence should be addressed to Dr. Eugene Tunik, Rutgers University, 65 Bergen Street, Room 714, Newark, NJ 07101. E-mail: eugene.tunik@rutgers.edu.

DOI:10.1523/JNEUROSCI.3000-14.2015

Copyright $\odot 2015$ the authors $\quad 0270-6474 / 15 / 352112-06 \$ 15.00 / 0$
}

previously (Borra et al., 2008), and neuronal activity in F5 is modulated by the type of grasp performed, suggesting a role in grip selection (Rizzolatti et al., 1988). In humans, PMv activation during grasping has been observed in imaging studies (Grafton et al., 1996; Binkofski et al., 1999). Paired-pulse TMS has been used to probe the influence of PMv over the motor cortex (M1) during grasping. Davare et al. $(2008,2009)$ demonstrated that PMv-M1 modulation tends to be inhibitory at rest but becomes excitatory in a grasp-specific manner upon preparation for a grasp, in line with our findings that TMS to PMv disrupts grasp planning (Tunik et al., 2008a). Notably, Buch et al. (2010) studied PMv-M1 interactions during grasp reprogramming for a visual perturbation of the target, noting that excitatory PMv-M1 modulation during grasp turns inhibitory during reprogramming in response to a visual perturbation of the goal. Together, these data support the notion that PMv may play a role in grip selection through the modulation of M1 when (re)planning grasping.

The above work highlights the involvement of the aIPS and PMv in updating grasp based on changes in visually defined goals. However, it remains unknown whether the PMv and aIPS likewise have roles in reprogramming for haptic perturbations of grasp. We directly test the causal involvement of the PMv and aIPS in reprogramming grip adjustments to a novel haptic finger perturbation during grasping. Subjects reached to grasp an object while having to overcome a continuous haptic perturbation extending the index finger, delivered on a minority of random trials. TMS was applied to the contralateral aIPS and PMv at 0, 50, 


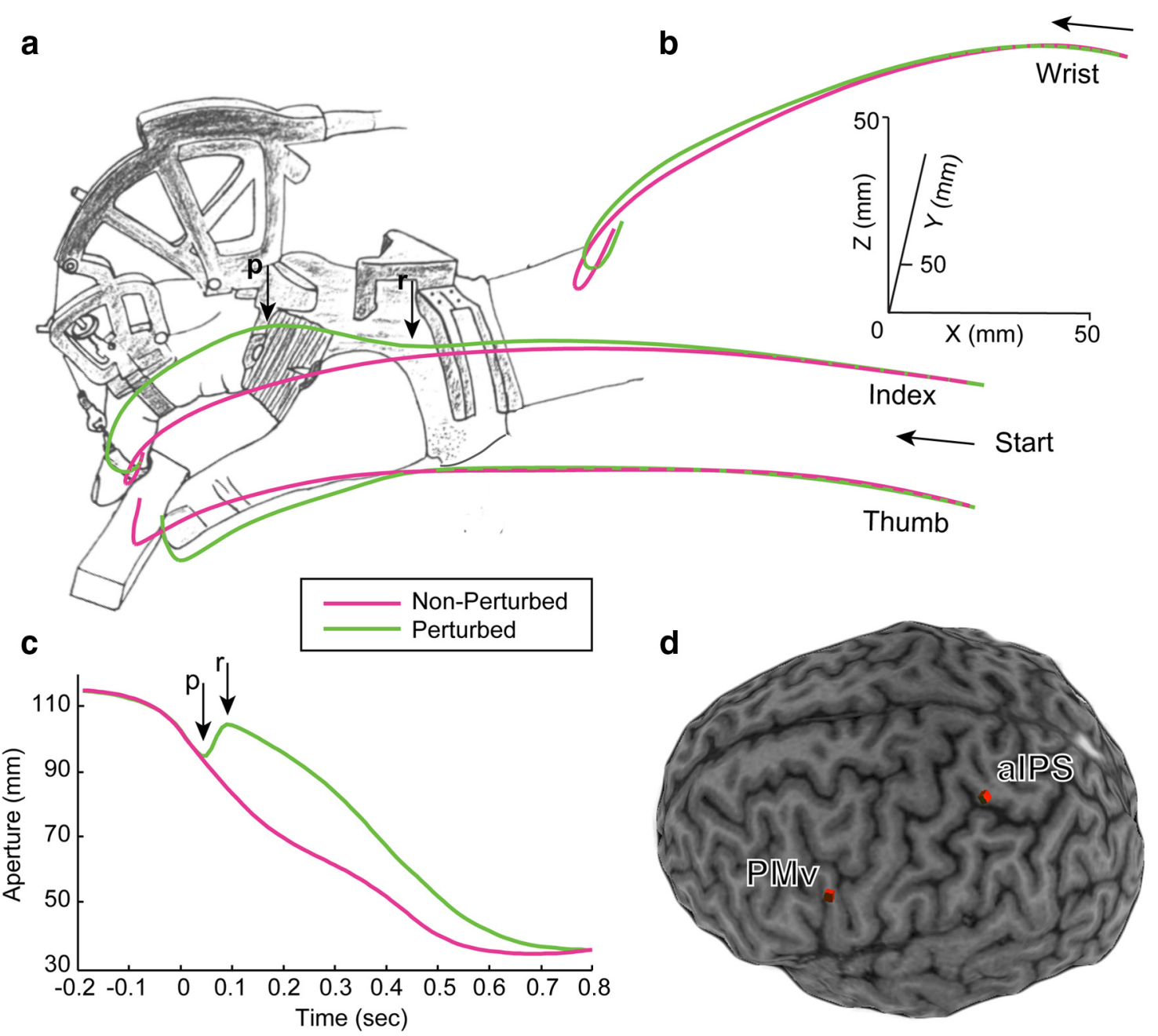

Figure 1. $\quad \boldsymbol{a}, \boldsymbol{b}$, Finger-wrist trajectories for No PERT and PERT trials in a typical subject. Note the continuous aperture reduction in No PERT trials (magenta) and a sudden widening of grasp in PERT trials (green) due to the haptic perturbation of the index finger. c, Aperture profiles for PERT and No PERT trials for the same subject. The $x$-axis is centered on the moment of the perturbation (time 0). The perturbation ( $\mathrm{p}$ ) resulted in a sudden increase of grasp aperture, a perturbation reversal $(r)$, and resumption of aperture closure. $\boldsymbol{d}$, PMv and alPS target sites.

or $100 \mathrm{~ms}$ following the perturbation to isolate the critical processing period. We hypothesized that if the aIPS or PMv is involved in reprogramming the action, TMS would disrupt grasping kinematics. Moreover, we expected that the latency at which TMS was applied would reveal whether these regions are involved in grip reprogramming in a serial manner. In other words, given the hypothesis that the aIPS may detect the perturbation and provide that information to the PMv, TMS to the aIPS should affect behavioral output at earlier latencies than when applied to the PMv.

\section{Materials and Methods}

Subjects. Ten right-handed healthy subjects (eight males; mean age \pm 1 $\mathrm{SD}, 30.5 \pm 6.6$ years old) participated after providing institutionally approved informed consent. Subjects were free of any orthopedic or neuromuscular diseases.

Setup. A force-producing exoskeleton (CyberGrasp) modified to reduce weight was donned over the right hand. Optical motion-capture sensors $(100 \mathrm{~Hz}$, Optotrak Certus, Northern Digital) were positioned on the tip of the thumb and index finger, and on the wrist. Seated subjects placed their hand flat on a table, with index finger and thumb tips 110 $\mathrm{mm}$ apart and the thumb pressing a trigger button.

Procedure. Following a "go" tone, subjects used a pincer grip (right hand) to reach to grasp a wooden block $(20 \times 20 \times 40 \mathrm{~mm})$ located 250 $\mathrm{mm}$ to the left and lift it $50 \mathrm{~mm}$ vertically at a natural pace (Fig. 1).
Subjects were given $2 \mathrm{~s}$ to complete the task and to return to the start. A random intertrial interval ( $3-5 \mathrm{~s})$ separated each trial.

Room lights were off during the movement, preventing vision of the hand and ensuring reliance on somatosensation to make on-line corrections to the perturbation. To prevent visual accommodation, lights were turned on between trials. The object glowed in the dark and was visible at all times.

Subjects performed 240 trials per block (aIPS- and PMv-TMS blocks). The block order was pseudorandom and counterbalanced. Twenty percent of trials ( 48 of 240) were "perturbed" (PERT), in which the CyberGrasp applied a sustained $0.5 \mathrm{~N}$ extension force to the index finger from the dorsal surface of the hand when the aperture was $<100 \mathrm{~mm}$. The apparatus produced no direct perturbation of the other fingers or hand. In the PERT condition, 12 trials involved no stimulation, whereas the rest involved stimulation (TMS delivered at 0,50 , or $100 \mathrm{~ms}, 12$ trials each, after the perturbation onset).

Since $80 \%$ of the trials were no-perturbation (No PERT) trials, subjects were unlikely to anticipate the perturbation. Of the 192 No PERT trials, 156 were stimulation trials, whereas the rest involved no TMS, with triggering the same as in the PERT condition. Therefore, the design was fully balanced with an equal number of TMS trials at each latency for PERT and No PERT conditions.

Neuronavigated transcranial magnetic stimulation. Subjects' MRIs (3T Siemens Allegra) were coregistered with their heads for frameless TMS neuronavigation (ANT Visor), ensuring consistency of stimulation location. Single-pulse TMS (Magstim Rapid2, $70 \mathrm{~mm}$ coil) was applied to 


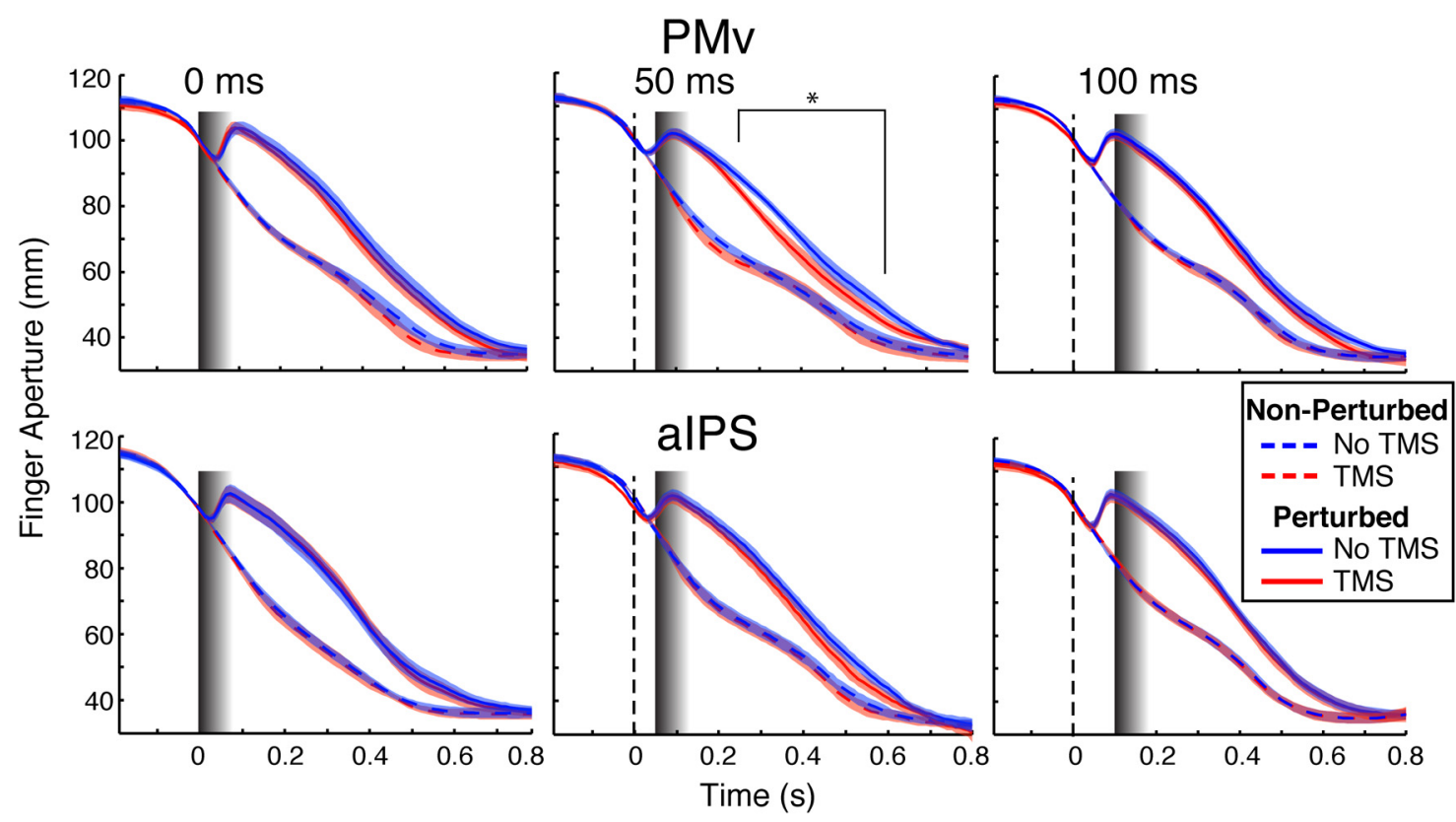

Figure 2. Group mean aperture traces ( $\pm 1 \mathrm{SE}$ ) for each condition in PMv (top) and alPS (bottom) blocks and TMS latency (columns). Black lines indicate the TMS onset. The gradient bars indicate the expected duration of TMS effects (Thut et al., 2003). Note that in TMS, the TMS effect overlaps with the onset of the perturbation; in TMS 50 , it overlaps with the perturbation reversal; and in TMS $_{100}$, it overlaps with the postreversal compensatory response. Red and blue lines represent the TMS and No TMS conditions, respectively. Solid and dashed lines represent the PERT and No PERT conditions, respectively. Asterisks ( $250-600 \mathrm{~ms}$ ) denote significant differences in aperture between TMS + PERT and No TMS + PERT conditions.

PMv [group mean (SD) MNI coordinates (mm) are as follows: $-50(3)$, 18 (4), 15 (3)] or aIPS [-42 (3), -37 (5), 49 (3); Fig. 2]. The loci were consistent with published work (PMv, $-58,13$, 19; aIPS, -40, -42, 46; Tunik et al., 2007; Davare et al., 2008, 2009). TMS intensity was $110 \%$ of resting motor threshold, defined per subject as the minimum intensity needed to elicit $>50 \mu \mathrm{V}$ motor-evoked potentials in the right first dorsal interosseous muscle on 5 of 10 consecutive trials.

Analysis. Kinematics were analyzed off-line using custom-written MATLAB (The MathWorks) scripts. For comparison across trials, PERT and No PERT movements were aligned in time according to when subjects' grasp aperture reached the perturbation threshold $(100 \mathrm{~mm})$. Since we were interested in the paths following the perturbation, trials were aligned in the vertical axis at the peak of the perturbation by obtaining the mean peak aperture of each subject's "PERT + No TMS" trials and aligning all "PERT + TMS" trials to it.

Each movement's onset and offset were identified as the time when grasp aperture fell and remained below $110 \mathrm{~mm}$ for $>50 \mathrm{~ms}$ and when it did not change by $>2 \mathrm{~mm}$ for $>100 \mathrm{~ms}$ (object grasp), respectively. The "movement time" was defined as the interval between the onset and offset. Kinematic traces were sampled in $50 \mathrm{~ms}$ intervals from 150 to 800 $\mathrm{ms}$ after the perturbation threshold (total 14 time points). We defined the "perturbation reversal" in PERT trials as the peak value of the grasp aperture following the onset of the perturbation.

Movement time and aperture reversal data were analyzed using separate three-way repeated-measures ANOVAs (RMANOVA) with the following factors (levels): Region (aIPS/PMv), Perturbation (Yes/No), and TMS Latency (Off/0 ms/50 ms/100 ms).

The grasp aperture for aIPS and PMv blocks was analyzed with separate three-way RMANOVAs: Perturbation (Yes/No), TMS Latency (Off/0 ms/50 ms/100 ms), and Time Point (150-800 ms). In cases where the sphericity assumption was violated, significance was established using Huynh-Feldt corrections. Significant three-way interactions were followed up with separate two-way RMANOVAs. Significant differences between conditions at specific time points were determined with Bonferroni-Holm sequential tests. The grip closure rate for the perturbation conditions was analyzed using a two-way RMANOVA: TMS Latency (Yes/No) and Time Point (150-800 ms).

\section{Results}

Figure 2 (top, middle) shows that TMS stimulation of the PMv 50 $\mathrm{ms}$ after the perturbation $\left(\mathrm{PMv}^{-\mathrm{TMS}_{50}}\right)$ resulted in a modification of the grasp aperture profile. A RMANOVA with Perturbation (Yes/No), TMS Latency (No/0 ms/50 ms/100 ms), and Time Point $(150-800 \mathrm{~ms})$ as factors was conducted on the aperture data for the PMv region. The Perturbation by Latency by Time Point interaction was significant $\left(F_{(39,351)}=2.91, p=0.02\right)$. A subsequent two-way RMANOVA for perturbed trials with TMS Latency (No/0 ms/50 ms/100 ms) and Time Point (150-800 ms) as factors revealed a significant interaction $\left(F_{(39,351)}=2.75, p=\right.$ 0.04). Post hoc pairwise comparisons at each time point showed significant differences between the aperture values in the TMS Latency $50 \mathrm{~ms}\left(\mathrm{PMv}^{-\mathrm{TMS}_{50}}\right)$ and No TMS conditions at time points $200-700 \mathrm{~ms}$ after perturbation $(p<0.01$ for time points 200,650 , and $700 \mathrm{~ms} ; p<0.001$ for time points $250-600 \mathrm{~ms}$ ). Notably, TMS stimulation at the three experimental latencies for aIPS did not lead to any significant differences between the TMS and No TMS traces (Perturbation by TMS Latency by Time Point interaction, $p>0.05)$.

To test the consistency of the PMv-TMS ${ }_{50}$ effect across subjects, we used paired $t$ tests to analyze the kinematic traces on a bin-by-bin basis within each subject (Fig. 3). In every subject, $\mathrm{PMv}_{\mathrm{TMS}}$ led to significant $(p<0.05)$ overestimation of grip aperture closure relative to the PERT + No TMS condition that persisted for a minimum of two consecutive $50 \mathrm{~ms}$ time bins (total interval, $100 \mathrm{~ms}$ ). This analysis confirms that the significant group-level effect is not driven by a subset of the data, but is rather a systematic effect in all subjects.

We next tested the dynamics of grasp aperture closure for the perturbation conditions in the PMv-TMS ${ }_{50}$ data to determine the timing of the compensatory response. For this, we conducted a RMANOVA on the rate of grip closure (aperture velocity; Fig. 4) for the perturbation conditions with factors TMS (On/Off) and 

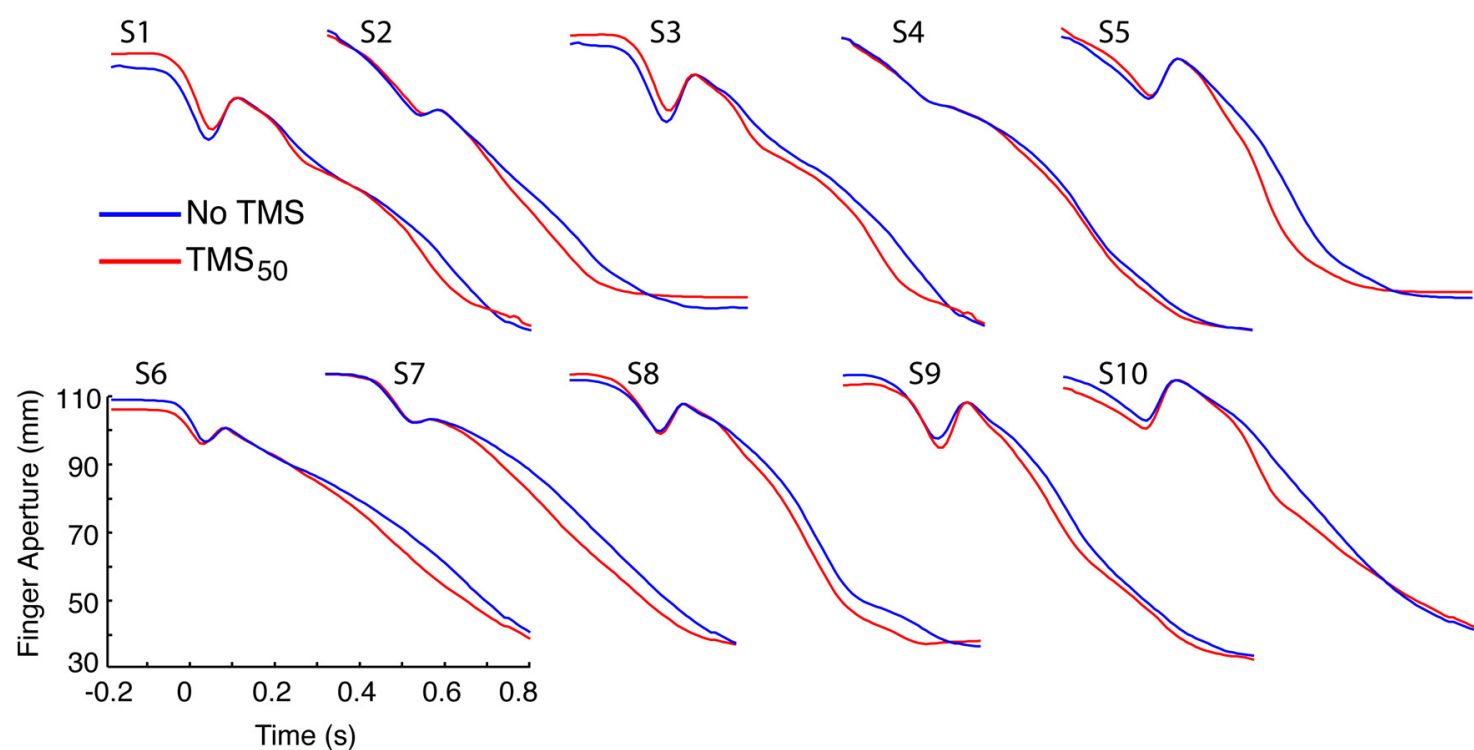

Figure 3. Mean aperture traces for PERT trials in each subject for the PMv-TMS ${ }_{50}$ condition. In each subject, TMS led to a significant overestimation of grasp aperture for at least two consecutive time bins (100 ms).
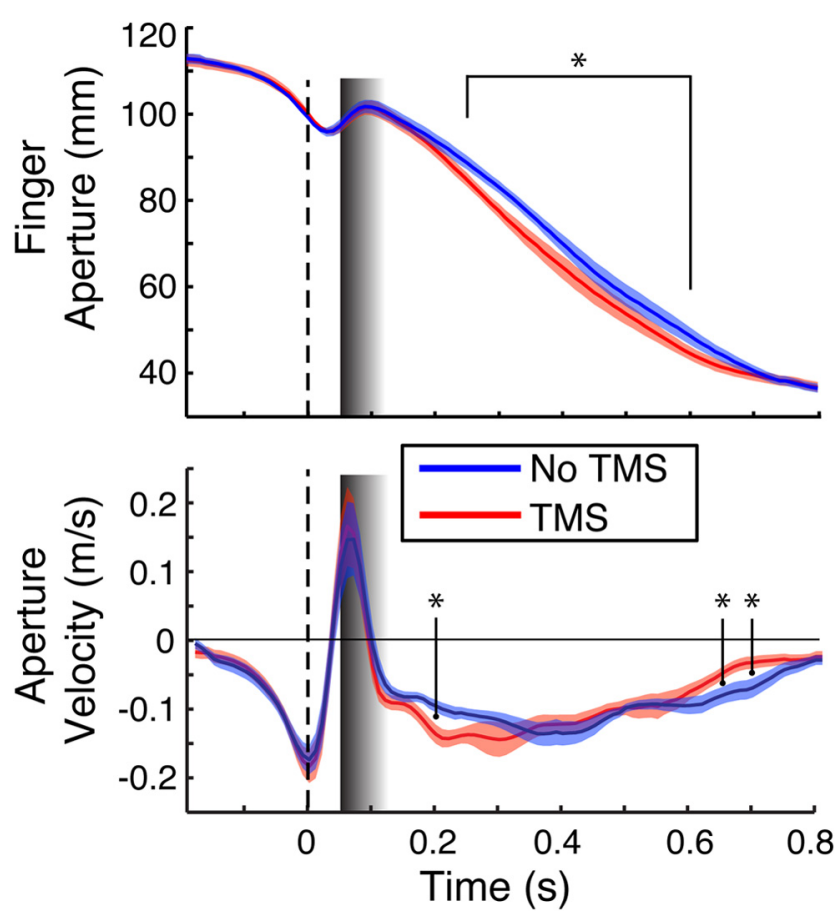

Figure 4. Group effect for $\mathrm{PMv}-\mathrm{TMS}_{50}$ for aperture (top) and rate of aperture closure (bottom) in the PERT condition. A significant increase in aperture closure rate at $200 \mathrm{~ms}$ (bottom, first asterisk) led to a modulation in aperture closure that persisted through the $250-600 \mathrm{~ms}$ interval (top). Consequently, the rate of aperture closure slowed toward the end of the movement in the TMS condition (bottom, last two asterisks) to prevent overclosing before object contact. All designations are as in Figure 2.

Time Point (150-800 ms). The RMANOVA revealed a significant main effect of Time Point $\left(F_{(13,117)}=9.83, p<0.0001\right)$ and a significant Time Point by TMS interaction $\left(F_{(13,117)}=5.27, p=\right.$ $0.009)$. Post hoc tests revealed significant differences between the two curves at time points $200 \mathrm{~ms}(p<0.0001), 600 \mathrm{~ms}(p=$ $0.0024), 650 \mathrm{~ms}(p=0.0027)$, and $700 \mathrm{~ms}(p=0.0043)$. These results indicate that subjects, following $\mathrm{PMv}^{-\mathrm{TMS}_{50}}$, responded to the perturbation by increasing the rate of grip closure at $\sim 200$ ms after the perturbation. Subsequently, toward the end of the movement, they stopped closing their grip altogether.

Given the significantly increased rate of closure, we tested whether TMS stimulation reduced subjects' time to complete the reach-to-grasp task. We conducted a RMANOVA on the total movement time with Region (aIPS/PMv), Perturbation (Yes/ $\mathrm{No})$, and TMS Latency (No/0 ms/50 ms/100 ms). Movement times never exceeded the $2 \mathrm{~s}$ maximum time allowed to complete the task. Expectedly, movement time was significantly longer in PERT trials than No PERT trials $\left(F_{(1,9)}=26.43, p<0.001\right.$; $1045 \pm 66 \mathrm{~ms}$ vs $950 \pm 76 \mathrm{~ms}$, respectively). However, no other main effects or interactions were noted $(p>0.05)$, suggesting that the initial overestimation of aperture closure in the PMv$\mathrm{TMS}_{50}$ condition was compensated for at the later stages of movement, perhaps to preserve coordination with transport.

To verify that the above observed effects were not attributable to the TMS-induced modulation of reaction time that has been reported in the literature (Day et al., 1989), we analyzed the time to perturbation reversal in PERT trials. A RMANOVA of the time to perturbation reversal with Region (aIPS/PMv) and TMS Latency $(\mathrm{No} / 0 \mathrm{~ms} / 50 \mathrm{~ms} / 100 \mathrm{~ms})$ as factors revealed no significant main effects or interactions (grand mean of time to perturbation reversal, $84.6 \pm 1.1 \mathrm{~ms})$.

\section{Discussion}

The present study shows that TMS-based disruption of cortical processing in the PMv results in modified grasp kinematics when subjects are required to correct for a haptic perturbation of finger aperture. The modified grasp kinematics are characterized by an increase in the speed of closure between the thumb and index finger occurring $\sim 200 \mathrm{~ms}$ after the perturbation, resulting in the early reduction of the distance between the fingers during the rest of the motion. Moreover, the effect was isolated to PMv-TMS delivered 50 $\mathrm{ms}$ (but not 0 or $100 \mathrm{~ms}$ ) after the perturbation, suggesting that cortical processing in the PMv for reprogramming the grasp occurred during a relatively short critical time window $\sim 50-100 \mathrm{~ms}$ after the perturbation. Conversely, aIPS-TMS did not result in significant changes at any of the three latencies used.

In previous work (Tunik et al., 2005), we showed that on-line grip reprogramming to compensate for a perturbation of a visu- 
ally defined target relied on the aIPS, and that the critical processing in the aIPS occurred approximately within $65 \mathrm{~ms}$ after the perturbation. Moreover, Rice et al., (2006) found that aIPS-TMS affects grip formation in the absence of a perturbation under certain circumstances, namely, whenever there is a need to determine whether the target object has changed; that is, aIPS processing appears not to be isolated strictly to the presence or absence of a visually defined perturbation of the movement goal, but rather responds to a need to update this information. Our current study answers a question we posed in Tunik et al. (2005) regarding the possibility that the aIPS may play a larger role when correcting for visually driven perturbations of a target goal compared to haptically defined perturbations, more specifically, that the aIPS may not be necessary for all forms of error correction, but rather may be specific for visually processed perturbations. Nonetheless, this result is also consistent with the idea that the aIPS may contain a somatosensory to visual gradient along its anteroposterior dimension involved in on-line correction to perturbations. Interestingly, whereas Gallivan et al. (2011) identify the anterior portion of the aIPS as being more related to somatosensory feedback, Reichenbach et al. (2014) suggest that the medial intraparietal sulcus (mIPS) is involved in processing proprioceptive feedback from the hand and may complement the visual functions of the aIPS. Future TMS studies using our paradigm should be able to address those contrasting findings.

Under our experimental conditions, it is important to carefully disentangle the processes that can be reasonably expected to play out during the detection of the perturbation and the execution of the motor response. Presumably, during the noperturbation conditions, the motor system selects an optimized habitual motor plan that includes an aperture-closing program with a relatively constant rate that is refined during the later phases of the grasp, as the fingers close on the target object (Schettino et al., 2003).

The haptic perturbation involves a sudden onset and continuous force from its onset to the end of the grasping movement. Therefore, the initial response to it requires subjects to detect and correct the unexpected extension and counteract the force applied to the finger before resuming aperture closure. This brief "perturbation reversal" process (Fig. 1) is critical for understanding the behavioral modification observed in our PMv-TMS condition.

Subjects reached the moment of the perturbation reversal with an average latency of $84.6 \pm 1.1 \mathrm{~ms}$. The short latency and small variability suggest that this fast reaction is most likely a triggered response akin to those observed in digit force modulation under unexpected loading paradigms (Johansson and Westling, 1988; Ehrsson et al., 2007). It has been suggested that this early reaction is mediated by a supraspinal loop involving primarily S1 and M1 and does not require modulation from posterior parietal or premotor cortices (Ehrsson et al., 2007; Pruszynski et al., 2014).

Although the initial response to the perturbation may be controlled by the sensorimotor strip, the fact that there is a behavioral goal defined in visual terms indicates the need for additional control processes. Subsequent to the perturbation reversal, the system must regulate the amount of force needed to balance out the steady pull of the apparatus and reorganize the grasping motion. Therefore, in our task, it seems reasonable to suggest that this critical information (needed to recalibrate the hand closure program) is not available until the perturbation reversal interval. This recalibration process is likely to require the involvement of parietal and/or premotor cortices to identify the amount of force needed to reprogram the closure program, especially given the unconstrained nature of the reach-to-grasp action.

An interesting finding in our study is that PMv-TMS at $50 \mathrm{~ms}$ in virtually all participants led to a systematic overestimation of the grip closure needed to counterbalance finger extension caused by the perturbation. Considering the generally accepted duration of cortical disruption produced by single-pulse TMS stimulation as lasting 50-100 ms (Thut et al., 2003), we can hypothesize that at this latency, our stimulation interfered with the computations needed to calibrate the closing of grip aperture by the motor system, rather than affecting the triggered response, which putatively occurred well before that. Moreover, the overestimation of grip aperture was unlikely due to low-level PMv-M1 TMS-induced facilitation because, if this were the case, we would expect to see similar effects in the other TMS conditions (e.g., for perturbed and unperturbed trials), and certainly in the $\mathrm{TMS}_{100}$ condition, since the TMS pulse in this condition immediately preceded the changes in aperture, neither of which was the case.

Instead, a likely possibility is that the PMv is needed during the perturbation for estimating the response magnitude required to attenuate the triggered response. Disrupting the PMv with TMS presumably interfered with this process, thereby allowing the triggered response to proceed too long, hence leading to an overestimated grip closure. Based on the notion that the PMv may play a regulatory role over $\mathrm{M} 1$ for reprogramming a movement following a perturbation (Buch et al., 2010), our contention is that the PMv-TMS ${ }_{50}$ pulse prevented the inhibitory control of M1 that was observed by Buch et al. (2010), resulting in the excess closure observed in our kinematic profiles during the grasp. In support of this notion, the subjects' aperture velocity profiles approached zero for the last $\sim 150 \mathrm{~ms}$ of the movement in the $\mathrm{PMv}^{-\mathrm{TMS}_{50}}$ condition, which was a necessary compensatory response given the excessive grip closure (Fig. 4).

Given the present results, we can surmise that the role of PMv in the response to a haptic perturbation could be (1) to determine the right amount of force and implement a new motor plan, (2) to implement the motor plan after being provided with the necessary information by another cortical region, and (3) to simply convey the information processed elsewhere to the motor cortex and/or spinal cord.

At present, we cannot distinguish among these possibilities because there are arguments to support all of them. Direct projections to the PMv from S1 have been reported in nonhuman primates (Stepniewska et al., 2006), suggesting that the PMv could receive somatosensory information directly from S1. More to the point, Bonnard et al. (2007) looked at the involvement of the PMv in the fine control of the pincer grip with fMRI and TMS. They found that as precision demands varied, the PMv exerted precise control of force during execution of the movement, indicating that the PMv may be sufficient to modulate grip aperture.

For the second possibility, as mentioned above, the mIPS may also receive somatosensory information related to the ongoing grasp and, parallel to visual perturbation processing by the aIPS, may in turn communicate with the PMv for the implementation of a new motor plan.

Finally, the PMv is known to have strong projections to both $\mathrm{M} 1$ and to the corticospinal tract, indicating that it could be just a link in the informational chain. We are currently pursuing these experimental hypotheses in our lab.

PMv-TMS pulses applied at the time of the perturbation $\left(\mathrm{PMv}^{-\mathrm{TMS}_{0}}\right)$ and $100 \mathrm{~ms}$ afterward $\left(\mathrm{PMv}^{-\mathrm{TMS}_{100}}\right)$ did not show any effects on the aperture profiles. In the case of $\mathrm{PMv}-\mathrm{TMS}_{0}$, the 
most parsimonious explanation is that at that latency, the PMv does not play a role in the organization and/or deployment of the triggered response. As mentioned above, triggered responses are thought to depend mainly on the sensorimotor strip for their control (Ehrsson et al., 2007). On the other hand, PMv-TMS ${ }_{100}$, which occurred after the moment of the perturbation reversal, may have disrupted PMv processing beyond the time at which the hand closure program needed for the reorganization of the grasp had been selected, eliminating the need for further reprogramming of the movement.

In summary, our data indicate that the aIPS may not play a critical role in the detection of grasping errors that are relayed through haptic means, despite the existence of a visually defined goal. Instead, our data suggest that the PMv is involved in reprogramming the grasp correction to a haptic perturbation during a transient "plastic" state (Tunik et al., 2008b) 50-100 ms after the perturbation.

\section{References}

Binkofski F, Buccino G, Stephan KM, Rizzolatti G, Seitz RJ, Freund HJ (1999) A parieto-premotor network for object manipulation: evidence from neuroimaging. Exp Brain Res 128:210-213. CrossRef Medline

Bonnard M, Galléa C, De Graaf JB, Pailhous J (2007) Corticospinal control of the thumb-index grip depends on precision of force control: a transcranial magnetic stimulation and functional magnetic resonance imagery study in humans. Eur J Neurosci 25:872-880. CrossRef Medline

Borra E, Belmalih A, Calzavara R, Gerbella M, Murata A, Rozzi S, Luppino G (2008) Cortical connections of the macaque anterior intraparietal (AIP) area. Cereb Cortex 18:1094-1111. CrossRef Medline

Buch ER, Mars RB, Boorman ED, Rushworth MF (2010) A network centered on ventral premotor cortex exerts both facilitatory and inhibitory control over primary motor cortex during action reprogramming. J Neurosci 30:1395-1401. CrossRef Medline

Culham J (2004) Human brain imaging reveals a parietal area specialized for grasping. In: Functional neuroimaging of visual cognition: attention and performance XX (Kanwisher N and Duncan J, eds.), pp 417-438. Oxford: Oxford UP.

Davare M, Lemon R, Olivier E (2008) Selective modulation of interactions between ventral premotor cortex and primary motor cortex during precision grasping in humans. J Physiol 586:2735-2742. CrossRef Medline

Davare M, Montague K, Olivier E, Rothwell JC, Lemon RN (2009) Ventral premotor to primary motor cortical interactions during object-driven grasp in human. Cortex 45:1050-1057. CrossRef Medline

Day BL, Rothwell JC, Thompson PD, Maertens de Noordhout A, Nakashima K, Shannon K, Marsden CD (1989) Delay in the execution of voluntary movement by electrical or magnetic brain stimulation in intact man. Evidence for the storage of motor programs in the brain. Brain 112:649663. CrossRef Medline

Ehrsson HH, Fagergren A, Ehrsson GO, Forssberg H (2007) Holding an object: neural activity associated with fingertip force adjustments to external perturbations. J Neurophysiol 97:1342-1352. Medline

Frey S, Vinton D, Norlund R, Grafton ST (2005) Cortical topography of human anterior intraparietal cortex active during visually guided grasping. Cogn Brain Res 23:397-405. CrossRef

Gallivan JP, McLean DA, Valyear KF, Pettypiece CE, Culham JC (2011) Decoding action intentions from preparatory brain activity in human parieto-frontal networks. J Neurosci 31:9599-9610. CrossRef Medline

Grafton ST (2010) The cognitive neuroscience of prehension: recent developments. Exp Brain Res 204:475-491. CrossRef Medline

Grafton ST, Fagg AH, Woods RP, Arbib MA (1996) Functional anatomy of pointing and grasping in humans. Cereb Cortex 6:226-237. CrossRef Medline

Johansson RS, Westling G (1988) Programmed and triggered actions to rapid load changes during precision grip. Exp Brain Res 71:72-86. Medline

Murata A, Gallese V, Luppino G, Kaseda M, Sakata H (2000) Selectivity for the shape, size, and orientation of objects for grasping in neurons of monkey parietal area AIP. J Neurophys 83:2580-2601. Medline

Pruszynski JA, Omrani M, Scott SH (2014) Goal-dependent modulation of fast feedback responses in primary motor cortex. J Neurosci 34:46084617. CrossRef Medline

Reichenbach A, Thielscher A, Peer A, Bülthoff HH, Bresciani JP (2014) A key region in the human parietal cortex for processing proprioceptive hand feedback during reaching movements. Neuroimage 84:615-625. CrossRef Medline

Rice NJ, Tunik E, Grafton ST (2006) The anterior intraparietal sulcus mediates grasp execution, independent of the requirement to update: new insights from transcranial magnetic stimulation. J Neurosci 26:81768182. CrossRef Medline

Rizzolatti G, Camarda R, Fogassi L, Gentilucci M, Luppino G, Matelli M (1988) Functional organization of inferior area 6 in the macaque monkey. II Area F5 and the control of distal movements. Exp Brain Res 71: 491-507. CrossRef Medline

Schettino LF, Adamovich SV, Poizner H (2003) Effects of object shape and visual feedback on hand configuration during grasping. Exp Brain Res 151:158-166. CrossRef Medline

Stepniewska I, Preuss TM, Kaas JH (2006) Ipsilateral cortical connections of dorsal and ventral premotor areas in New World owl monkeys. J Comp Neurol 495:691-708. Medline

Thut G, Northoff G, Ives JR, Kamitani Y, Pfennig A, Kampmann F, Schomer DL, Pascual-Leone A (2003) Effects of single-pulse transcranial magnetic stimulation (TMS) on functional brain activity: a combined event-related TMS and evoked potential study. Clin Neurophysiol 114:2071-2080. CrossRef Medline

Tunik E, Frey SH, Grafton ST (2005) Virtual lesions of the anterior intraparietal area disrupt goal-dependent on-line adjustments of grasp. Nat Neurosci 8:505-511. Medline

Tunik E, Rice NJ, Hamilton A, Grafton ST (2007) Beyond grasping: representation of action in human anterior intraparietal sulcus. Neuroimage 36 [Suppl 2]:T77-T86. Medline

Tunik E, Lo OY, Adamovich SV (2008a) Transcranial magnetic stimulation of the frontal operculum and supramarginal gyrus disrupts planning of outcome-based hand-object interactions. J Neurosci 28:14422-14427. CrossRef Medline

Tunik E, Ortigue S, Adamovich SV, Grafton ST (2008b) Differential recruitment of anterior intraparietal sulcus and superior parietal lobule during visually guided grasping revealed by electrical neuroimaging. J Neurosci 28:13615-13620. CrossRef Medline 